\title{
Geriatric Intensive Care Unit Admission Protocol
}

Tom Byvoets ${ }^{1}$, Brian Brewer ${ }^{2}$

${ }^{1}$ Indiana University School of Medicine; ${ }^{2}$ Indiana University School of Medicine, Department of Surgery

\section{Background and Hypothesis:}

The Society of Critical Care Medicine has recommended protocolized admission and discharge criteria for the ICU, but there is little supporting evidence for these guidelines. Our goal is to find predictors supported by data that can consistently triage patients appropriately on admission and decrease the need to upgrade the level of care during the same hospital stay. We hypothesized that applying a protocol would reduce the number of unplanned ICU admissions in our institution.

\section{Methods:}

We retrospectively applied our protocol to patients sixty-five years or older from the trauma registry at Methodist Hospital admitted between January 1 and December 31, 2019. Patients who did not have an unplanned ICU admission during hospital stay were used as the control group, while those who had an unplanned ICU admission event were considered the experimental group. After this, we used crosstabulation and Fisher's exact test to analyze the data.

\section{Results:}

We reviewed one thousand one hundred forty-six (1146) subjects with 1088 in the control group and 58 in the experimental group. Subjects in the experimental group were statistically more likely to have COPD (OR: 2.646, $p=0.001$ ), been transfused $>$ two units of PRBC in the first 24 hours (OR: 5.303, $p=0.000$ ), or to have greater than two rib fractures (OR: 2.469, $p=0.002$ ).

\section{Conclusion and Potential Impact:}

This study demonstrates that applying this protocol can capture elderly patients who will likely require ICU level care during their hospitalization. COPD, > two rib fractures, and transfusion of $>$ two units PRBC during the first 24 hours appear to be significant predictors of clinical deterioration. Unplanned ICU admissions are associated with increased hospital and ICU length of stay. We must develop evidence-based protocols to decrease morbidity and cost associated with unplanned ICU admissions. 